Method We used a minimally-invasive endovascular EC collection technique to obtain aneurysmal ECs from aneurysm coils and access sheaths during endovascular treatment. Cells were collected from unruptured and ruptured aneurysms with lowand high-risk PHASES scores, as well as control arteries. After fluorescent-activated cell sorting (FACS), cytoplasmic mRNA from individual cells were extracted with SmartSeq Single Cell $^{\mathrm{TM}}$ and cDNA libraries were constructed for single cell RNA-Seq.

Results Non-deployed endovascular coils and access sheaths from 15 patients (8 unruptured and 7 ruptured aneurysms) with a median PHASES score of 7 , ranging from $3-11$, were safely collected during endovascular treatment without adverse event. Cells were dissociated from the hardware and viable endothelial cells were identified by FACS with CD31 antibodies in 8 patients $(5$ unruptured and 3 ruptured aneurysms. Extracted RNA from single cells produced $>500$ pg of cDNA which was used for cDNA library construction and submitted for RNA sequencing.

Conclusion Endovascular sampling of unruptured and ruptured aneurysm is safe and feasible. Despite the dissociation and FACS processes, endothelial cells were viable and produced sufficient cDNA at a single cell level for the construction of cDNA library for RNA sequencing. We envisage that this technique will greatly enhance the availability of cellular material for genetic study of cerebrovascular diseases.

Disclosures C. Young: 1; C; AANS/CNS CV Section Robert J Dempsey Award 2019/2020, Bee Foundation Aneurysm Research Grant. S. Levy: None. A. Patel: None. D. Cooke: None. Y. Zheng: None. C. Mandrycky: None. C. Kelly: None. L. Kim: None. M. Levitt: None.

\section{E-015 BEYOND THE WALL: IMAGING OF PERIVASCULAR STRUCTURES OF THE INTRACRANIAL SUBARACHOID SPACE}

V Anagnostakou*, Z Vardar, A Puri, G Ughi, M Gounis. Radiology, University of Massachusetts, Worcester, MA

\subsection{6/neurintsurg-2020-SNIS.51}

Introduction A new method of intravascular imaging is introduced, high-frequency optical coherence tomography (HFOCT), with resolution unprecedented for in vivo imaging $(\sim 10 \mu \mathrm{m})$. Here we describe the first preclinical observations of cerebrovascular imaging with this new technology.

Methods Two dogs were imaged using HF-OCT. A 6F straight guiding catheter was initially positioned into the right vertebral artery up to the origin of the right lateral spinal ramus anastomoticus and the basilar artery was accessed with an SL-10 microcatheter. In the first dog access was achieved through the main lumen of the vertebral artery coursing along the vertebral column around the C1 vertebral body and finally the vertebrobasilar junction and the basilar artery. In the second $\operatorname{dog}$ access was achieved through the right lateral spinal ramus and the anterior spinal artery, the diamond shaped confluence of anastomotic channels between vertebral arteries and spinal rami into the basilar artery. In both animals the microcatheter was advanced up to the P1P2 segment of the left PCA in order to facilitate positioning of the HF-OCT wire which was unsheathed from the microcatheter. Using contrast at a flow rate of $4 \mathrm{ml} / \mathrm{sec}$ to wash out the blood in the vessels imaging of the basilar artery from distal to proximal was performed. 
Results Detailed images of the basilar artery, lower cerebellar arteries, basilar perforators, vertebrobasilar junction, spinal perforators were obtained (figure 1). Due to the high resolution of the method, the layers of the vessel wall were imaged. What was even more interesting was the ability to image beyond the artery into the structural components of the subarachnoid space with the arachnoid membranes and what we believe to be the arachnoid trabeculae. Venous structures and nerve root origins were also recognized.

Conclusion HF-OCT imaging can offer detailed visualization not only of the vessel wall, the branching pattern of basilar and spinal perforators but also of structures of the subarachnoid space impossible to study with other imaging modalities due to inadequate resolution. The clinical implication of such an analysis is yet to be discovered.

Disclosures V. Anagnostakou: None. Z. Vardar: None. A. Puri: None. G. Ughi: None. M. Gounis: None.

\section{E-016 A MODIFIED RABBIT ELASTASE INDUCED ANEURYSM THAT SHOWS CONTINUED INFLAMMATION AND GROWTH}

1,2R King*, '1 Caroff, 'A Leopardi, ${ }^{1} \mathrm{~A}$ Bogdanov, ${ }^{1} \mathrm{M}$ Gounis. ${ }^{1}$ Radiology, University of Massachusetts Medical School, Worcester, MA; ${ }^{2}$ Biomedical Engineering, Worcester Polytechnic Institute, Worcester, MA

\subsection{6/neurintsurg-2020-SNIS.52}

Introduction Animal models of saccular aneurysms are stable following creation, and do not actively remodel or rupture. Recently, rodent models of aneurysm wall remodeling and rupture have been described by surgical grafting of decellularized arterial tissue. ${ }^{1}$ We sought to 1) develop and apply a modified in situ decellularization approach, not require complex grafting procedures, in a large animal model of saccular aneurysms and 2) investigate modified aneurysmal expansion and inflammation using in vivo imaging.

Methods 18 New Zealand White rabbits (3.0-3.5 kg) underwent standard elastinolysis aneurysm creation of the right common carotid artery. The animals were randomized

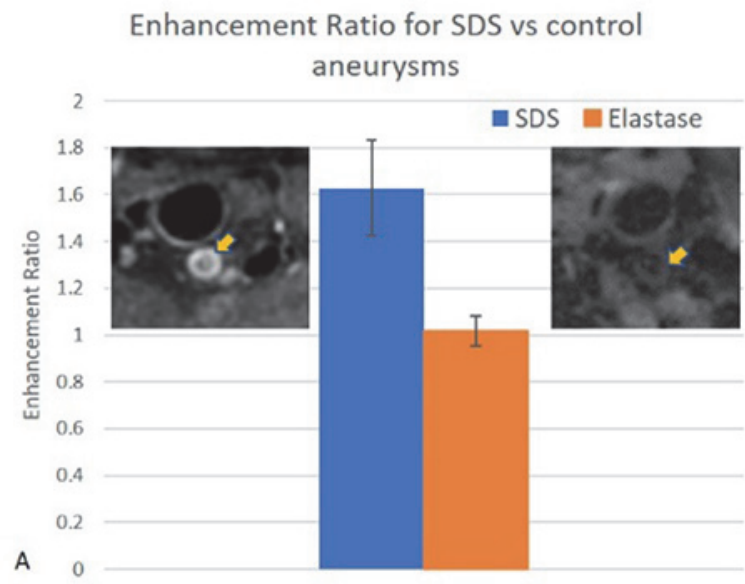

2:1 to receive isolated vessel SDS treatment (perfusion with $1 \%$ solution for $45 \mathrm{~min}$ ) or nothing. Every two weeks until week 8 , three of the rabbits (2 SDS+elastase, 1 elastase), were subjected to MR imaging, ${ }^{2}$ followed by contrast injection with stable macrocyclic gadolinium(III)-based contrast agent that has been shown to selectively enhance the areas of vessel wall myeloperoxidase (MPO) accumulation. ${ }^{3}$ 4 The MRI was repeated at 3 hours post contrast injection and the enhancement ratio (ER) was calculated. All aneurysms were measured for size by digital subtraction angiography.

Results Of the 17 rabbits included in the study, 11 were treated in situ with SDS following elastase incubation and 6 served as controls. One SDS-treated rabbit was excluded due to rupture of the carotid artery during recovery. During follow-up MR imaging the ER was greater than 1 for all SDStreated animals, whereas the controls had an ER of greater than 1 only at the two-week timepoint (consistent with the expected healing process). Average ER for SDS-treated animals was $1.63 \pm 0.20$, while control animals had an ER of 1.02 $\pm 0.06(\mathrm{p}<0.001)$. SDS-treated aneurysms width increased by over $45 \%$ over the first 8 weeks, while the control aneurysms grew about $20 \%$ in the first 2 weeks and then remained stable.

Conclusions During the 12 weeks post aneurysm creation, MPO-mediated MR signal enhancement was continuously detected within the wall of the aneurysm, and other than at 2 weeks, only in the SDS-treated animals. This novel modified large animal aneurysm model is expected to be useful for testing of new therapies that rely on not only the mechanical properties of the aneurysm but also the biological processes that make aneurysms vulnerable to rupture.

\section{REFERENCES}

1. Stroke 2014;45:248-254.
2. AJNR 2015;36:146-52.
3. Radiology 2009;252:696-703.
4. Sci Rep 2018;8:7687.

Disclosures R. King: None. J. Caroff: 1; C; FullBright. A. Leopardi: None. A. Bogdanov: None. M. Gounis: None.

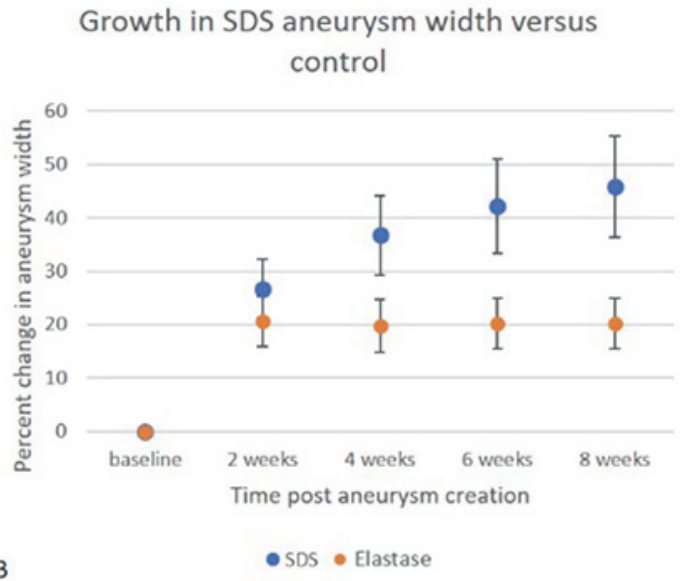

Abstract E-016 Figure 1 A) overall enhancement ration of SDS vs control aneurysms, showing a significant difference $(p<0.001)$, The left subpanel shows an SDS treated aneurysm (arrow) after contrast injection, right subpanel shows a control aneurysm (arrow) after contrast injection. B) Growth in the width of the aneurysm width over time. After two weeks, the control aneurysm stabilize in size, while the SDS aneurysms continue to grow. Due to reduction in numbers at each time point, standard deviation increases at each follow up timepoint 